NASA/TM-2006-214320

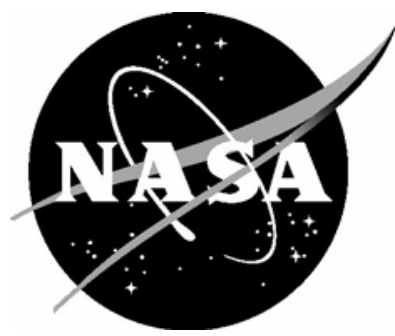

\title{
A Wireless Fluid-Level Measurement Technique
}

Stanley E. Woodard

Langley Research Center, Hampton, Virginia

Bryant D. Taylor

Swales Aerospace, Hampton, Virginia 
Since its founding, NASA has been dedicated to the advancement of aeronautics and space science. The NASA Scientific and Technical Information (STI) Program Office plays a key part in helping NASA maintain this important role.

The NASA STI Program Office is operated by Langley Research Center, the lead center for NASA's scientific and technical information. The NASA STI Program Office provides access to the NASA STI Database, the largest collection of aeronautical and space science STI in the world. The Program Office is also NASA's institutional mechanism for disseminating the results of its research and development activities. These results are published by NASA in the NASA STI Report Series, which includes the following report types:

- TECHNICAL PUBLICATION. Reports of completed research or a major significant phase of research that present the results of NASA programs and include extensive data or theoretical analysis. Includes compilations of significant scientific and technical data and information deemed to be of continuing reference value. NASA counterpart of peerreviewed formal professional papers, but having less stringent limitations on manuscript length and extent of graphic presentations.

- TECHNICAL MEMORANDUM. Scientific and technical findings that are preliminary or of specialized interest, e.g., quick release reports, working papers, and bibliographies that contain minimal annotation. Does not contain extensive analysis.

- CONTRACTOR REPORT. Scientific and technical findings by NASA-sponsored contractors and grantees.
- CONFERENCE PUBLICATION. Collected papers from scientific and technical conferences, symposia, seminars, or other meetings sponsored or co-sponsored by NASA.

- SPECIAL PUBLICATION. Scientific, technical, or historical information from NASA programs, projects, and missions, often concerned with subjects having substantial public interest.

- TECHNICAL TRANSLATION. Englishlanguage translations of foreign scientific and technical material pertinent to NASA's mission.

Specialized services that complement the STI Program Office's diverse offerings include creating custom thesauri, building customized databases, organizing and publishing research results ... even providing videos.

For more information about the NASA STI Program Office, see the following:

- Access the NASA STI Program Home Page at http://www.sti.nasa.gov

- E-mail your question via the Internet to help@sti.nasa.gov

- Fax your question to the NASA STI Help Desk at (301) 621-0134

- Phone the NASA STI Help Desk at (301) 621-0390

- Write to: NASA STI Help Desk NASA Center for AeroSpace Information 7121 Standard Drive Hanover, MD 21076-1320 
NASA/TM-2006-214320

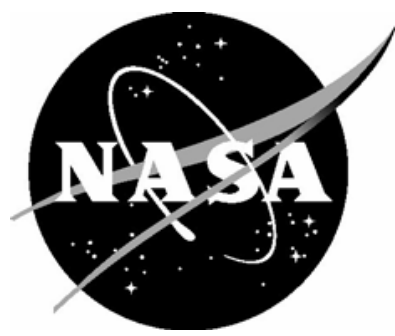

\section{A Wireless Fluid-Level Measurement Technique}

Stanley E. Woodard

Langley Research Center, Hampton, Virginia

Bryant D. Taylor

Swales Aerospace, Hampton, Virginia

National Aeronautics and

Space Administration 
Available from:

NASA Center for AeroSpace Information (CASI)

7121 Standard Drive

Hanover, MD 21076-1320

(301) 621-0390
National Technical Information Service (NTIS) 5285 Port Royal Road Springfield, VA 22161-2171

(703) 605-6000 


\title{
A Wireless Fluid-Level Measurement Technique
}

\author{
Stanley E. Woodard and Bryant D. Taylor \\ NASA Langley Research Center \\ Hampton, VA 23681
}

This paper presents the application of a recently developed wireless measurement acquisition system to fluid-level measurement. This type of fluid-level measurement system alleviates many shortcomings of fluid-level measurement methods currently being used, including limited applicability of any one fluid-level sensor design. Measurement acquisition shortcomings include the necessity for power to be supplied to each sensor and for the measurement to be extracted from each sensor via a physical connection to the sensor. Another shortcoming is existing measurement systems require that a data channel and signal conditioning electronics be dedicated to each sensor. Use of wires results in other shortcomings such as logistics needed to add or replace sensors, weight, potential for electrical arcing and wire degradations. The fluidlevel sensor design is a simple passive inductor-capacitor circuit that is not subject to mechanical failure that is possible when float and lever-arm systems are used. Methods are presented for using the sensor in caustic, acidic or cryogenic fluids. Oscillating magnetic fields are used to power the sensor. Once electrically excited, the sensor produces a magnetic field response. The response frequency corresponds to the amount to fluid within the capacitor's electric field. The sensor design can be modified for measuring the level of any fluid or fluent substance that can be stored in a non-conductive reservoir. The interrogation method for discerning changes in the sensor response frequency is also presented. 


\section{Introduction}

Existing methods of measuring fluid levels are based upon capacitance, hydrostatic pressure, visual inspections (e.g., viewing glass in wall of container or manual dipstick immersion into and retrieval from a container), mechanical floats, ultrasonics and fiber optics. The aforementioned methods have been used in many industrial applications for which the specific use of each one is suitable. However, all have limitations when considered for a broad range of use in extreme environments such as cryogenic, acidic, caustic or with high temperature fluids. Electromechanical fluid-level measurement systems such as those that use floats must take into consideration weight and volume of the mechanical system. Visual inspection is not feasible for automatic or autonomous systems. In cases where the fluid vapors should not be released into the atmosphere, visual inspections requiring that the fluid container lid be open are not appropriate.

Ultrasonic methods of measuring fluid levels are non-invasive [1]. All measurements can be discerned external to a container. Ultrasonic methods are not suitable if gas bubbles are in the fluid or the containers do not have surfaces with good acoustic reflections. Furthermore, containers with internal structures such as baffles can scatter acoustic waves[1]. Ultrasonic measurements are not feasible for reservoirs within outer walls such as double hull oil tankers or aircraft nose landing gear. Measurement error can result from acoustic signal variation due to change in the gas/vapor density above the fluid being measured. Highly mechanically damped substances limit the use of these methods.

Fiber optic methods are inherently safe and can be used in combustible liquids because the light propagated through the fiber is modulated to correspond with a liquid level instead of modulating electical voltage [2-6]. The fibers themselves are not susceptible to electromagnetic interference. However, the support acquisition equipment is susceptible to electromagnetic interference. The fiber optic sensors are not suitable for fluids that leave a film residue (e.g., a viscous fluids or sludge) nor can they be used in caustic fluids, powders, grainular substances or cryogenic fluids.

This paper presents a fluid-level sensor designed as a magnetic field response sensor. Magnetic field response sensors are a class of sensors that are powered via oscillating magnetic fields and when electrically active respond with their own magnetic fields whose attributes are dependent upon the physical properties being measured [7]. Refs. [7-12] describe a measurement acquisition method for powering and acquiring measurements from magnetic field response sensors. The method is simple to use and has many advantages over other methods currently in use [7]. The fluid level sensor is a passive resonant capacitance-inductance circuit. The circuit capacitor can either be capacitive plates or interdigital electrodes. When the capacitor is immersed in a fluid, its capacitance will change proportional to the area of the capacitor plates (or number of interdigitated electrode pairs) exposed to the fluid. The circuit's resonant frequency is dependent upon the capacitance resulting in a direct correlation between the circuit's resonant frequency and the amount of capacitor exposure to the fluid. Faraday induction via harmonic magnetic fields is used to produce an electromotive force and therefore a current in the circuit [13-14]. An antenna electrically coupled to an amplitude modulated current produces a time-varying magnetic field used to power the sensor. Once electrically active, the circuit produces its own harmonic magnetic field as the inductor stores and releases magnetic 
energy. The magnetic field produced by the inductor is received by an antenna. The antenna is not physically connected to the sensor resulting in a method of providing power to the sensor and acquiring the measurement from the sensor without the use of wires or cables. The fluid-level sensor powered and interrogated via the magnetic field response measurement acquisition method is a wireless technique for measuring the level of any fluent substance (e.g., liquid, amorphous substance, grain or powder) for which the electrical dielectric is constant.

The advantage that the wireless fluid-level measurement technique presented herein has over the other methods is that the sensors are electrically passive and do not need physical or electrical connections to a power source or measurement acquisition equipment. Furthermore, the measurement technique used does not necessitate a dedicated measurement channel for each sensor [7]. The sensors can be used in conditions such as caustic environments, acids, high temperatures, cryogenic temperatures and high pressures. Sensors and the supporting measurement acquisition hardware can be lightweight and non-obtrusive. Many components of the method can be integrated with structural components of a system. Results will be presented that demonstrate the use of the sensors on a viscous fluid, liquid nitrogen and other fluent substances. Eliminating wiring for acquiring measurements can alleviate potential hazards associated with wires. Wiring hazards include damaged wires becoming ignition sources due to arcing. Wire damage can result from wire fraying, chemical degradation of wire insulation, wire splaying and wire chaffing (e.g., under a clamp). Many of the aforementioned methods of wire damage have resulted in critical spacecraft launch delays or aircraft fatalities [15-18].

The simplest embodiment of a magnetic field response fluid-level sensor is that of two capacitive plates coupled to an inductor [19]. Another embodiment is that of a thin-film sensor having a spiral trace inductor electrically coupled to interdigitated electrodes [8 and 12]. The interrogation method presented in [19] requires close proximity $(<5 \mathrm{~cm})$ to the sensor. This paper presents a method that facilitates interrogation of either of the aforementioned sensors at distances greater than $60 \mathrm{~cm}$ using the measurement acquisition method discussed in [7]. Furthermore, methods are presented that show how the sensor design of [19] can be modified for measuring the level of any fluid (including caustic or acidic) that can be stored in a nonconductive reservoir or for measuring any non-conductive fluid.

Following this introduction will be a discussion of the magnetic field response measurement acquisition method used to supply power to the sensors and to acquire the measurement from the sensors. The fluid-level sensor will be presented next. The discussion of the sensor includes a mathematical model of the sensor that can be used to design its range of frequency response and measurement sensitivity. Sensitivity to key design parameters is also presented. Characterization results from using the sensors for a variety substances including liquid nitrogen will be presented. Adapting the sensors for high temperature, caustic, corrosive and cryogenic environments will be presented next followed by a discussion of fluid film residue on the sensor. 


\section{Magnetic Field Response Acquisition Method}

The acquisition method presented herein is applicable to any passive inductive-capacitive resonant circuit such as the fluid-level sensors discussed in this paper. This section will discuss measurement acquisition for any magnetic field response sensor. A detailed discussion of the method is given in Ref. [7]. The next section will discuss a fluid-level sensor that can be powered and interrogated using the measurement acquisition method presented in this section.

Refs. [19-21] discuss several methods of inductive-capacitive sensor interrogation. The interrogation method discussed in Ref [19] uses a receiver that consists of an amplifier circuit with an inductor coil connected to the amplifier input and another inductor coil connected to the amplifier output. The coils are wound and are positioned with respect to each other to balance mutual inductance such that feedback between the coils is approximately zero. The balancing objective is to not create a positive input voltage to the amplifier. A voltage is applied to the amplifier thus powering the output inductor. When the fluid-level sensor is placed in proximity to the two coils, its inductor causes the amplifier output inductor to oscillate at the sensor's resonant frequency. The amplifier output inductor will cause the amplifier input inductor to oscillate thus creating a feedback system. The input to the amplifier is then correlated to a value of fluid level. The limitation of this interrogation method is that the prefered separation distance between the sensor and the interrogator is no more than $3.5 \mathrm{~cm}$. Another limitation of the method used in Ref. [19] is that it only interrogates the frequency of the sensor. This is sufficient when only one physical property is to be measured using the same sensor. Should simultaneous measurements of two physical properties using the same sensor be required, it would be necessary to measure response frequency and either response amplitude or bandwidth.

Various interrogations methods are presented in Ref. [21]. One method uses a transmitting antenna coupled to an impedance analyzer. The sensor's magnetic field response creates a change in the frequency spectrum of the transmitted signal. The transmitting antenna's electrical current peaks at the sensor resonant frequency. The change is used to ascertain the physical property being measured. In another method, Ref [21] using a transmission of white noise to power the sensor. A receiving antenna is electromagnetically coupled to the transmission antenna. The receiving antenna receives the transmission antenna signal negated by the sensor response in a manner similar to a dip meter. The last method discussed in [21] is that of broadcasting white noise for a predetermined period of time. The transmission stops and the sensor response is interrogated. In all aforementioned methods, the sensors are within close proximity to the antennae $(<10 \mathrm{~cm})$ used for interrogation. . The interrogation method presented in this paper has produced an interrogation distance of over $3 \mathrm{~m}$ using two antenna and $1 \mathrm{w}$ of power and $0.6 \mathrm{~m}$ using a single antenna power with $0.1 \mathrm{w}$. The measurement system presented in this paper is capable of examining the sensor response amplitude and frequency. Ref. [7] describes how the sensor bandwidth can be inferred.

Fig. 1 shows a schematic of the measurement acquisition method for magnetic field response sensors using a single antenna and multiple magnetic field response sensors. The components of the measurement system are an antenna for transmitting and receiving magnetic field energy; a processor for regulating the RF transmission and reception; software for control of the antenna 
and for analyzing the signals received; and, magnetic field response sensors. The processor modulates the input signal to the antenna to produce either a broadband time-varying magnetic field or a single harmonic magetic field. The variable magnetic field creates an electrical current in the passive inductor-capacitor circuits as a result of Faraday induction. The circuits will electrically oscillate at resonant electrical frequencies that are dependent upon the capacitance and inductance of each circuit. The oscillation occurs as the energy is harmonically transferred between the inductor (as magnetic energy) and capacitor (as electrical energy). When the energy is in the inductors, the magnetic fields produced are single harmonic radio frequencies whose frequencies are the respective circuits resonant frequencies. The antenna is also used to receive the harmonic magnetic responses produced by the inductors of the circuits. The receiving antenna can be the same antenna used to produce the initial broadcast of energy received by the circuit or another antenna can be used. When the same antenna is used, it must be switched from a transmitting antenna to a receiving antennna. A simple microprocessor can be used to identify the frequencies of the signals received by the antenna. The measured frequencies are then correlated to measurement of physical states.

The interrogation method is that of a "transmit-receive-compare" technique [7]. A single harmonic is transmitted from the transmission antenna. The sensor is electrically excited thus causing its inductor to produce a magnetic harmonic. A receiving antenna receives the sensors magnetic field response. The response amplitude and frequency are stored. The current amplitude is then compared to the previous one. Each sensor has a dedicated range of frequencies (measurement bands). A single antenna sweep covers measurement bands for all sensors. A sweep is a series of increasing magnetic field harmonics. Sensor measurement bands and the sampling resolution for $\mathrm{n}$ sensors are shown in Fig. 2 for successive frequency sweeps. Each sensor has a frequency resolution $(\Delta \omega)$ that corresponds to a measurement resolution of the physical property. The sweep of individual frequencies is used because it concentrates all energy used to excite the sensor at a single frequency producing a high signal-to-noise ratio. The upper graphic in Fig. 2 depicts the sensor response amplitude as the excitation frequency approaches the sensor resonant frequency.

Separate transmission and receiving antennae can be used or a single switching antenna can be used. Using two antennae provides a larger volumetric swath at which measurements can be taken that is approximately double that of a single antenna. The interrogation procedure is as follows:

1. At the lower limit of a predetermined range, a magnetic field harmonic is transmitted for a predetermined length of time and then the transmission mode is switched off (i.e., the transmission antenna is turned off if two antennae are used or if a single antenna is used, it ceases transmission).

2. The receiving mode is then turned on (i.e., the receiving antenna is turned on if two antennae are used or if a single antenna is used, it begins receiving). The received response from the sensor is rectified to determine its amplitude. The amplitude, $A_{i}(t)$, and frequency, $\omega_{i}(t)$, are stored in memory. 
3. The receiving mode is turned off and the transmission mode is turned on. The transmitted magnetic field harmonic is then shifted by a predetermined amount (i.e., based upon sensor frequency resolution). The harmonic is transmitted for a predetermined length of time and then the transmission mode is turned off.

4. The receiving mode is then turned on. The received response from the sensor is rectified to determine it amplitude. The amplitude, $A_{i}$, and frequency, $\omega_{i}$, are stored in memory.

5. The current amplitude, $A_{i}$, is compared to the two previously stored amplitudes, $A_{i-1}$ and $A_{i-2}$. If the previous amplitude, $A_{i-1}$, is greater than the current amplitude, $A_{i}$, and if the previous amplitude is greater than amplitude prior to it, $A_{i-2}$, the previous amplitude, $A_{i-1}$, is the peak amplitude. The peak amplitude occurs when the excitation harmonic is equal to the resonant frequency of the sensor. The amplitude, $A_{i-1}$, and the corresponding frequency, $\omega_{\mathrm{i}-1}$, are cataloged for the sensor for the current frequency sweep. These values can be compared to the values aquired during the next sweep. If an amplitude peak has not been identified, then steps 3 and 4 are repeated.

6. If amplitude peak has been identified, the harmonic sweep continues to the next sensor.

Basically, the objective of the aforementioned method is to identify the inflection point of the each sensor's magnetic field response amplitude. The frequency at which the amplitude peak occurs is the resonant frequency. Once peak amplitude has been detected, the peak amplitude and frequency are stored and then the next partition is examined. After the last partition is examined, a new sweep is started. The initial frequency sweep can be used to identify and catalog all resonant amplitudes and frequencies associated with all sensors within the antenna's spatial and spectral range of interrogation. The spatial range of interrogation is discussed in Refs. [9] and [12]. The sweep duration must less than half the Nyquist period of the measured physical state with the highest frequency. For example, if one sensor is measuring vibrations of less than $30 \mathrm{~Hz}$ and other measured states have rates of change less than $30 \mathrm{~Hz}$, then the sweeps must be done at a rate of $60 \mathrm{~Hz}$ or greater. The cataloged amplitudes and resonant frequencies for all sensors can be used to reduce the sweep time for successive sweeps. The next sweep to update each resonant frequency can start and end at a predetermined proximity (i.e., narrowing the measurement range) to the cataloged resonant and then skip to the next resonant (Fig. 2). Narrowing subsequent sweep bands could be used as a means of increasing the sweep rate. Not all measurement bands need to be interrogated for successive sweeps. If a physical state does not change rapidly or is somewhat quasi-static, it may not be necessary to collect its measurement at each sweep. In Fig. 2, only three sensors are interrogated for the ith sweep.

Dynamic measurements can also be produced by comparing variation in frequencies and amplitudes of the current sweep with those of the prior sweeps. An example is that of a sensor having fixed inductance and capacitance (i.e., frequency will remain constant) moving with respect to a stationary antenna. As the sensor moves with respect to the antenna, the amplitudes for successive sweeps will be different. (e.g., sensor is placed on a moving object like a rotating wheel). The change in circuit position and rate of position change can be determined by comparing the amplitudes $\left(\mathbf{A}_{n}\right)$ variations of successive sweeps (as shown by the third last sensor in Fig. 2). The magnitude and sign of the difference can be used to determine how fast the 
sensor is moving and whether the sensor is moving toward the antenna or away from the antenna. Another example would be that of a magnetic field response fluid-level sensor in a reservoir that is being filled. The rate that the reservoir is being filled can be determined by comparing the frequencies of successive sweeps.

The sensor responses are superimposed. The sensors are designed (Fig. 2) such that their range of measurement frequencies do not overlap but the ranges are within a frequency spectrum of the antenna. The range of resonant frequencies for each sensor corresponds to physical property values that can be measured. An example would be that the lower frequency in the measurement band, $\omega_{i}(p)$, would correspond to the lower limit of a strain measurement. This method allows for multiple sensors within the spatial and spectral range of the antennae to be interrogated. It should be noted that the discrete frequencies need not be evenly spaced in any measurement band. The higher the number of discrete frequencies within a band, the higher sensor resolution.

The acquisition method provides a means for powering passive inductive-capacitive sensors and acquiring measurements from them without having a physical connection to a power source or to acquisition hardware. Multiple sensors can be interrogated using a single data acquisition channel when a switching transmitting/receiving antenna used. Inductors, antennae and some capacitor types can be designed as thin metallic foils or thin deposited metallic films. No lineof-sight is required between antenna and sensors. Many designs of magnetic field response sensors can be embedded in non-conductive material. For conducting material, the capacitive element can be embedded and the inductive element can be placed away from the surface of the conductive material. No specific orientation of the sensor with respect the antenna is required except that they cannot be 90 deg to each other.

Because the sensors do not need a physical connection to a power source or data acquisition equipment, they are easy to implement into existing vehicles, structures or other existing systems. All that is required to add a new measurement is to have the sensor within the interrogation antenna(s)' magnetic field. No wiring is required. Each sensor must have a dedicated frequency partition of the antenna spectrum. The interrogation system must have for each sensor, a table that correlates one or more magnetic field response attributes with a one or more physical states being measured.

A hand-held version of the interrogation system using the acquisition method discussed in this section is the magnetic field response recorder shown in Fig. 3. The programmable recorder is designed to be simple to use and is capable of powering and acquiring measurements from any magnetic field response sensor. It can acquire and interpret measurements that correspond to either the magnetic field response sensor's amplitude, frequency and/or bandwidth. Each sensor's resonant response frequency and amplitude are displayed. The amplitude is dependent upon distance at which the sensor is interrogated [7]. The recorder can be programmed to display the physical value of the measurement. The magnetic field response recorder has an internal antenna, connector for external antenna and an analog output. Results of using the recorder will be presented later in this paper. 


\section{Method of Acquiring Fluid-Level Measurements}

A magnetic response fluid-level sensor requires two components electrically connected. The first component is that of a capacitor whose electric field can be variably exposed to a fluid. The second component is an inductor that stores and releases magnetic energy at a harmonic rate being that of the resonant frequency of the circuit formed by the inductor and capacitor. For simplicity of discussion, a fluid-level sensor consisting of two parallel electroplates electrically connected to a lamina spiral inductor will be used to describe the method of measuring fluid level. Fig. 4 shows the sensor with key geometric parameters annotated. Although a lamina spiral is shown, other inductor designs can be used. The direction of electric field is shown also. Fig. 4 depicts a sensor completely immersed in two non-mixing stratified dielectric substances. A substance (e.g., a fluid) of dielectric constant, $\kappa_{2}$, fills the lower portion of the gap between the electric plates to level $z$. The remaining portion of the gap is filled with a substance of dielectric constant, $\kappa_{1}$. When air is the substance in the upper portion of the capacitor, $\kappa_{1} \approx 1$. The density of $\kappa_{2}$ is far greater than that of $\kappa_{1}$ and $\kappa_{2}>>\kappa_{1}$. The capacitance, $C(z)$, is dependent upon the dielectric interface level, $z$, and is the summed capacitance due to contributions from both dielectric substances. When the upper dielectric is air, the sensor serves has a fluid-level sensor. The two portions of the capacitor act as parallel capacitors since they share the same electric field. The sensor capacitance, $C(z)$, is

$$
\begin{aligned}
C(z) & =C_{\kappa 1}+C_{\kappa 2} \\
& =(\ell-z) \frac{\varepsilon_{0} w \kappa_{1}}{d}+\frac{\varepsilon_{0} w \kappa_{2}}{d} z \\
& =\frac{\varepsilon_{0} w}{d}\left[\ell \kappa_{1}+\left(\kappa_{2}-\kappa_{1}\right) z\right]
\end{aligned}
$$

$C_{\kappa 1}$ and $C_{\kappa 2}$ are the capacitance contributions due to $\kappa_{1}$ and $\kappa_{2}$, respectively. The key geometric parameters of this embodiment are total length of electroplates, $\ell$; width of the electroplates, $w$; separation of the electroplates, $d$; and, the dielectric constants, $\kappa_{2}$ and $\kappa_{1}$. The permittivity constant, $\varepsilon_{0}$, is $\left(8.85 \times 10^{-12} \mathrm{~F} / \mathrm{m}\right)$. When the space between the capacitor plates is completely filled with dielectric $\kappa_{1}$ (i.e., $z=0$ ), the capacitance is

$$
C(z)=\frac{\varepsilon_{0} w \ell \kappa_{1}}{d}
$$

The capacitor gap completely filled with $\kappa_{2}$ (i.e., $z=\ell$ ) has capacitance of

$$
C(z)=\frac{\kappa \varepsilon_{0} w \ell \kappa_{2}}{d}
$$


The resonant electrical frequency of the circuit is

$$
\omega=\frac{1}{2 \pi \sqrt{L C(z)}}
$$

Inclusion of the equation for capacitance, Eq. (1), into that for resonant frequency, Eq. (2), results in the following expression which relates the resonant frequency to the dielectric interface level, $z$, (fluid level when upper dielectric is air)

$$
\omega=\frac{1}{2 \pi}\left[\frac{L \varepsilon_{0} w \ell}{d}\left[\kappa_{1}+\left(\kappa_{2}-\kappa_{1}\right) \frac{z}{\ell}\right]\right]^{-1 / 2}
$$

There is a unique frequency, $\omega$, for any level that is bounded by two extrema, $\omega_{U}$ and $\omega_{L}$.

with

$$
\omega_{U}>\omega>\omega_{L}
$$

$$
\omega_{U}=\frac{1}{2 \pi}\left[\frac{L \varepsilon_{0} w \ell \kappa_{1}}{d}\right]^{-1 / 2} \text { and } \omega_{L}=\frac{1}{2 \pi}\left[\frac{L \varepsilon_{0} w \ell \kappa_{2}}{d}\right]^{-1 / 2}
$$

The upper frequency value, $\omega_{U}$, corresponds to the sensor's capacitive plates being completely immersed in dielectric $\kappa_{1}$ and lower frequency value, $\omega_{L}$, corresponds to the sensor's capacitive plates being completely immersed in dielectric $\kappa_{2}$.

Supplying power to the sensor and acquiring the measurement from the sensor can be done using the method discussed in the previous section. The measurement acquisition method can be used to acquire measurements from multiple sensors. When other fuel-level sensors are placed within the same reservoir, each sensor can be distinguished by designing the frequency range, $\omega_{U}>\omega>\omega_{L}$, of the sensors so that they do not overlap. Designing the frequency range can be done by selecting capacitor and inductor physical dimensions.

The first derivative of Eq. (3) with respect to the interface level, $z$, can be used to understand how the physical properties of the sensor affect measurement sensitivity. The derivative is

$$
\frac{d \omega}{d z}=-\frac{\left(\kappa_{2}-\kappa_{1}\right)}{4 \pi}\left[\frac{L \varepsilon_{0} w \ell}{d}\right]^{-1 / 2}\left[\kappa_{1}+\left(\kappa_{2}-\kappa_{1}\right) \frac{Z}{\ell}\right]^{-3 / 2}
$$


The terms in the first bracket are those that would be primary design parameters. These terms are of order $\mathrm{O}(-1 / 2)$. The measurement sensitivity increases with decreased inductance, plate width and plate length. The sensitivity increases with increased electroplate separation. The sensitivity also increases with a larger difference in dielectric values. The terms in the second bracket are of order $\mathrm{O}(-3 / 2)$. The first term is dependent upon the dielectric of the upper substance. The second term is dependent upon the difference between the dielectric values and what fraction of the sensor is exposed to the fluid. The sensitivity is dependent upon the dielectric interface level and is variable between two extrema. The extrema occur when the sensor has no exposure to the dielectric and when the sensor is completely exposed to the dielectric. Eqs. (3) and (4) are applicable for sensors whose capacitor electric fields approximate being evenly spaced and are not dominated by having electric field lines fringing at the edges. The effect of fringing will be more pronounced as the size of either the width and length are near or less than the plate separation. Eqs. (3) and (4) also do not account for any parasitic inductance or capacitance in the sensor.

\section{Measured Sensor Response Frequency to Dielectric Immersion Level}

The previous sections discussed a non-contact method of powering an inductive-capacitive circuit and how such a circuit could be developed to measure a fluid level. This section will discuss results of measuring fluid level using two sensor designs. The first design consists of two Bakelite strips at a fixed separation with respect to each other. Copper foil is adhered to the two strip surfaces facing each other. Non-conductive washers are placed between to the strips to maintain a fixed separation. The strips are electrically connected to a square spiral inductor with an inductance of $49.8 \mu \mathrm{H}$. The first magnetic field response fluid-level sensor is shown in Fig. 5. The sensor was designed to allow its geometry to vary. Capacitor plate spacing could be changed by the number of washers used. Copper foil was used to allow the plate length and width to be progressively cut away to reduce the sensor area.

The capacitor was placed in a graduated cylinder while the inductor remained outside the container. The container was filled with transmission fluid (dielectric constant of 2.2 at $27 \mathrm{deg}$ C). As the fluid filled the void between the plates, the dielectric exposure increased proportional to fluid immersion thus changing the sensor's resonant frequency. Combinations of capacitor plate widths and lengths used are shown in Table 1. Three measurements were performed for each combination. Capacitor plate spacing was fixed at $0.32 \mathrm{~cm}$. Fluid level was increased using $13 \mathrm{~mm}$ increments. During each measurement, the interrogation antenna was maintained at a fixed distance from the sensor inductor. The results of response frequency variations with fluid level are shown in Fig. 6 for the capacitor geometric combinations given in Table 1. The measurement sensitivity decreased with increasing length for the same value of width and increased with increasing width for the same value of length. Because of the sensor geometry (i.e., the sensor width being equal to or less than the capacitor plate spacing), electric field fringing at the edges influenced the measurements and sensitivity of the sensor. 


\begin{tabular}{|c|c|c|c|}
\hline Length (cm) & Width $(\mathrm{cm})$ & $\Delta$ Frequency $(\mathrm{MHz})$ & $\begin{array}{c}\text { Response Frequency } \\
\text { Change with Fluid } \\
\text { Level }\end{array}$ \\
\hline 7.62 & 0.16 & 0.569 & -0.190 \\
\hline 7.62 & 0.32 & 0.950 & -0.317 \\
\hline 15.24 & 0.16 & 0.796 & -0.134 \\
\hline 15.24 & 0.32 & 1.077 & -0.180 \\
\hline 22.86 & 0.16 & 0.876 & -0.097 \\
\hline 22.86 & 0.32 & 1.153 & -0.128 \\
\hline
\end{tabular}

Table 1 Sensor frequency response sensitivity to length and width

Because sensor response is based upon the amount and type of dielectric material placed within the capacitor's electric field, the sensor is capable of measuring the level of any fluent substance. In the design shown in Fig. 5, any non-conductive dielectric would produce a similar set of the sensitivity curves shown in Fig. 6. The measurements presented thus far were those of a viscous liquid.

The second wireless fluid-level sensor developed is shown in Fig. 7. A flat 7.62 by $6.35 \mathrm{~cm}$ spiral $45 \mu \mathrm{H}$ inductor is placed in a housing. Both are mounted on top of a capacitor frame. The inductor is electrically connected to two capacitor plates each with lengths of $25.90 \mathrm{~cm}$. Plate separation is $0.32 \mathrm{~cm}$. The inductor housing protects the inductor from its environmental and potential damage. Both the inductor housing and the capacitor frame are made of Ertalyte ${ }^{\circledR}$ PET-P which is developed by Quadrant Engineering Plastics. The plastic has dimensional stability, high strength, resistance to moderately acidic solutions and continuous service temperature of $210^{\circ} \mathrm{F}\left(100^{\circ} \mathrm{C}\right)$. It can be used in cryogenic environments and is capable of sustaining high loads and enduring wear conditions. Ertalyte has good chemical and abrasion resistance. Furthermore Ertalyte has low moisture absorption that enables mechanical and electrical properties to remain virtually unaffected by moisture. Also shown in Fig. 7 is a magnetic field response recorder. During the interrogation of a sensor, the antenna shown need not be in contact with the sensor or its housing.

Liquid nitrogen, a powder, and a granular substance were chosen to demonstrate the capability of the sensor and the interrogation system shown in Fig. 7 for measuring different fluent substances. Liquid nitrogen is non-viscous and cryogenic. Repeated measurements of liquid nitrogen level demonstrated potential use for the sensor in other liquid cryogens like liquid oxygen and hydrogen. Liquid nitrogen has a dielectric constant of approximately 1.4. A dewar and the sensor are shown in Fig. 8, along with the antenna that was placed on top of the inductor housing to maintain a constant separation with respect to the inductor. The antenna is connected to a magnetic field response recorder (Fig. 7b) via the cable connected to the antenna. The funnel was used to assist in replenishing the liquid nitrogen level. Replenishment was necessary due to outgassing. During testing, the sensor was placed in a full Dewar of liquid nitrogen and then removed at increments of $1.3 \mathrm{~cm}$. At each increment, the sensor was interrogated. Once completely removed, the sensor was immersed into the Dewar in $1.3 \mathrm{~cm}$ increments. Two 
sensors $(0.32 \mathrm{~cm}$ and $0.63 \mathrm{~cm}$ width plates $)$ were tested. The tests were repeated at minimum of three times for each sensor.

Results of testing the sensors with the Ertalyte inductor housing and capacitor frame are shown in Fig. 9. Total immersion of the sensor capacitor plates into the liquid nitrogen were approximately $16 \mathrm{~cm}$. Three immersion and removal cycles are shown for each sensor. The difference between the maximum and minimum values for any specific level for all three cycles were less than or equal to $0.05 \mathrm{MHz}$ which was equivalent to $14 \%$ error. Between tests, it was necessary to replenish the liquid nitrogen level because of outgassing. In some cases, the level had been reduced by $1.3 \mathrm{~cm}$. Hence, the error can be mostly attributed to reduction in the total liquid level due to outgassing during the testing. These preliminary tests demonstrated that the sensors are capable of measuring liquid cryogens.

The other fluent substances measured were sugar (dielectric constant of 1.5-2.2) and ground corn (dielectric constant of 2.3-2.6). The ground corn was used to demonstrate that the sensor could be used for granular-type substances whose size would not prevent them from being placed between the capacitor plates. Similarly, cane sugar was used because it was a fine powder. Results of measuring the various dielectrics (sugar, liquid nitrogen, ground corn and transmission fluid) are shown in Fig. 10. The sensor, Fig. 7, with the 0.32 wide electroplates was used for all measurements shown in Fig. 10. The measurement curve for liquid nitrogen is at a higher initial value than that of the other substances due to its lower temperature. The approximate slope is larger for substances of higher dielectric constant. The curves corroborate the measurement sensitivity given in Eq. (4).

\section{Method of Acquiring Fluid-Level Measurements in Harsh Environments}

The previous section presented measurement results that demonstrated that the measurement method presented in this paper is viable to many fluent substances. In this section, a sensor design is presented that extends the functionality of the previous designs to that of facilitating the measurement of substances (e.g., acid) that would normally destroy electronic circuitry or measurements within environments that are capable of destructing many electronic circuits (e.g., high temperature, caustic, etc.) The sensors presented in this paper and the method for powering them and interrogating them allow the sensors to be completely encased in materials that are resilient to the aforementioned environments. In essence, a fluid-level sensor can be produced that does not require any of its electrical components to be in physical contact with the substance that is being measured. For example, the encasing material could be a plastic chemically resilient to acids; a material capable of sustained cryogenic exposure for liquid nitrogen, hydrogen, helium or oxygen; a material of low thermal conduction for molten metals; or, containing a large number of hydrogen nuclides for radiative environments. The design shown in Fig. 7 demonstrated that the inductor could be encased and if necessary hermetically sealed. The entire sensor could be encased and hermetically sealed to prevent any degradation to sensor due to exposure to caustic gases. This section extends the analysis presented in the section "Method of Acquiring Fluid-Level Measurements" to examine how the sensor's magnetic field response is influenced by encasing material thickness and dielectric constant. The other sensor 
parameters previously discussed (inductance, capacitor plate spacing, length and width, and the dielectric properties of the fluent substance being measured) are included.

Two capacitor plates encased in a material with dielectric constant, $\kappa_{\text {case }}$, are shown in Fig. 11. The sensor shown in Fig. 7 could be modified such that its electric plates are encased as shown in Fig 11 thereby having the entire sensor encased. Similar to Eq. (1), the capacitance, $C_{e}(z)$, is dependent upon the dielectric interface level, $z$, and is the summed capacitance due to exposure to both dielectric substances (e.g., air and gasoline). However, the capacitance is also dependent upon the casing dielectric properties and thickness. To understand the influence of the capacitor casing, it is necessary to know the electric field in the encasing material and the fluid. Refs. 1 and 2 provide detailed discussions of electric fields and capacitance. The portion of Fig 11 having the fluid is shown in Fig. 12. Fig. 12 only shows the plates and the dielectrics between the plates. The casing material affixed to the outside surface of the plates does not influence the measurement response because the electric fields are only within the plates. The two plates are shown with the left plate having a charge of $+q$ and the right plate having a charge of $-q$. The electric field when no dielectric is present is

$$
E_{0}=\frac{q}{\varepsilon_{0} A}
$$

The charges, $+q$ and $-q$, are produced from the electromotive force produced as result of the oscillating magnetic field generated by the antenna. The area, $A$, is the product of the capacitor width, $w$, and the length, $z$, of the portion of the sensor exposed to the fluid. An encasing material of thickness, $d_{\text {case }}$, and dielectric constant, $\kappa_{\text {case }}$, is placed on each capacitor plate within the electric field. Because the material is dielectric, the electric field, $E_{0}$, produces polarized charges $+q^{\prime}$ and $-q^{\prime}$ that are distributed on the outer surface of the encasing material. These charges are shown in Fig. 12. The electric field between the plates, $E_{0}$ is reduced within the material proportional to the dielectric constant. Within the encasing material the electric field, $E_{\text {case }}$, is

$$
E_{\text {case }}=\frac{E_{0}}{\kappa_{\text {case }}}=\frac{q}{\kappa_{\text {case }} \varepsilon_{0} A}
$$

In a similar manner, polarized charges $+q^{\prime \prime}$ and $-q^{\prime \prime}$ are distributed on the outer surfaces of the fluid between the encasing material. The electric field within the fluid, $E_{f l}$ is that of $E_{0}$ proportionally reduced by the fluid's dielectric constant, $\kappa_{f l}$. Therefore

$$
E_{f l}=\frac{E_{0}}{\kappa_{f l}}=\frac{q}{\kappa_{f l} \varepsilon_{0} A}
$$


The potential difference across the sensor plates is

$$
\begin{aligned}
V & =-\int_{0}^{d} E \cdot d \ell \\
& =-\int_{0}^{d} E \cos \left(180^{\circ}\right) d \ell \\
& =\int_{0}^{d} E d \ell \\
& =E_{\text {case }} d_{\text {case }}+E_{f l}\left(d-2 d_{\text {case }}\right)+E_{\text {case }} d_{\text {case }} \\
& =\left(\frac{q}{\varepsilon_{0} A}\right)\left[\frac{2 d_{\text {case }}}{\kappa_{\text {case }}}+\frac{\left(d-2 d_{\text {case }}\right)}{\kappa_{f l}}\right] \\
& =\left(\frac{q}{\varepsilon_{0} A \kappa_{\text {case }} \kappa_{f l}}\right)\left[2 d_{\text {case }} \kappa_{f l}+\left(d-2 d_{\text {case }}\right) \kappa_{\text {case }}\right]
\end{aligned}
$$

The integral is taken from the negative plate to the positive plate. The contribution, $C_{f l}$, to the sensor capacitance due to the portion of the sensor that is immersed in the fluid is

$$
\begin{aligned}
C_{f l} & =\frac{q}{V} \\
& =\frac{\varepsilon_{0} A \kappa_{\text {case }} \kappa_{f l}}{2 d_{\text {case }} \kappa_{f l}+\left(d-2 d_{\text {case }}\right) \kappa_{\text {case }}}
\end{aligned}
$$

The area exposed to the fluid is $A=z w$. The portion of the sensor below $z$ contributes the following to the total capacitance

$$
C_{f l}=\frac{\varepsilon_{0} Z w \kappa_{\text {case }} \kappa_{f l}}{2 d_{\text {case }} \kappa_{f l}+\left(d-2 d_{\text {case }}\right) \kappa_{\text {case }}}
$$

Eq. (10) shows that the dielectrics within the plates act as three capacitors $\left(\frac{\varepsilon_{0} A \kappa_{\text {case }}}{d_{\text {case }}}, \frac{\varepsilon_{0} A \kappa_{f l}}{\left(d-2 d_{\text {case }}\right)}\right.$ and $\frac{\varepsilon_{0} A \kappa_{\text {case }}}{d_{\text {case }}}$ ) in series. In the portion of the sensor not exposed to the fluid, $A=(\ell-z) w$ and $\kappa_{f l} \approx 1$. The contribution, $C_{0}$, to the total capacitance for that portion of the sensor is 


$$
C_{0}=\frac{\varepsilon_{0}(\ell-z) w \kappa_{\text {case }}}{2 d_{\text {case }}+\left(d-2 d_{\text {case }}\right) \kappa_{\text {case }}}
$$

The total sensor capacitance, $C_{e}$ is

$$
\begin{aligned}
C_{e}(z) & =C_{0}+C_{f l} \\
& =\frac{\varepsilon_{0}(\ell-z) w \kappa_{\text {case }}}{2 d_{\text {case }}+\left(d-2 d_{\text {case }}\right) \kappa_{\text {case }}}+\frac{\varepsilon_{0} z w \kappa_{\text {case }} \kappa_{f l}}{2 d_{\text {case }} \kappa_{f l}+\left(d-2 d_{\text {case }}\right) \kappa_{\text {case }}}
\end{aligned}
$$

Eq. (12) is continuous with respect to fluid level. The resonant frequency of the circuit when an inductor is electrically connected to the capacitance is

$$
\omega_{e}(z)=\left[\frac{L \varepsilon_{0}(\ell-z) w \kappa_{\text {case }}}{2 d_{\text {case }}+\left(d-2 d_{\text {case }}\right) \kappa_{\text {case }}}+\frac{L \varepsilon_{0} z w \kappa_{\text {case }} \kappa_{f l}}{2 d_{\text {case }} \kappa_{f l}+\left(d-2 d_{\text {case }}\right) \kappa_{\text {case }}}\right]^{-1 / 2}
$$

The inductor can be placed within the same housing as the capacitor so that the completed circuit is contained within one housing.

When the capacitor gap is completely filled with fluid, the sensor response frequency is the lower bound of its frequency range and is

$$
\omega_{e}(\ell)=\left[\frac{L \varepsilon_{0} \ell w \kappa_{\text {case }} \kappa_{f l}}{2 d_{\text {case }} \kappa_{f l}+\left(d-2 d_{\text {case }}\right) \kappa_{\text {case }}}\right]^{-1 / 2}
$$

The response frequency when the sensor is completely empty is the upper bound of its frequency range and is

$$
\omega_{e}(0)=\left[\frac{\varepsilon_{0} \ell w \kappa_{\text {case }}}{2 d_{\text {case }}+\left(d-2 d_{\text {case }}\right) \kappa_{\text {case }}}\right]^{-1 / 2}
$$

To understand the influence of the casing on the measurement, one must examine the capacitance of Fig. 12 as the casing thickness diminishes (i.e., the limit for $C_{e}$ as $d_{\text {case }} \rightarrow 0$ ). As the casing thickness diminishes, $\kappa_{\text {case }} \rightarrow 1$ above $Z$ and $\kappa_{\text {case }} \rightarrow \kappa_{f l}$ below $z$. As the thickness diminishes, Eq. (12) reduces to Eq. (1) when the upper fluid in Figs. 4 and 11 is air. The effect of the casing is that the sensor's resonant frequency range will be shifted. The amount of the 
shift is dependent upon the casing thickness and material. Similar to the previous design, the sensor's resonant frequency will vary with the amount of capacitor plate area exposed to the fluid. Therefore, a sensor with its electrical components (inductor, capacitor and electrical connections between them) could be completely encased. The encased sensor can receive power via Faraday induction and produce a magnetic field response whose frequency is dependent upon fluid level.

\section{Effect of Residual Viscous Film on Sensor Measurement}

Many viscous substances leave a film residue on the electroplates when removed. When use of the sensor prevents cleaning between measurements, it is necessary to know the effect that dielectric residue has on measurement error. This section will discuss how the sensor response is affected by any residue left on a sensor after a measurement. Fig. 13 illustrates electroplates with a separation distance, $d$. A film of thickness, $b$, is left of each plate. The separation of the plates is far greater than the thickness of the film (i.e., $b<<d$ ).

The voltage across the electroplates is dependent upon the electric field through the dielectric, $E_{b}$, and the free air, $E_{0}$.

$$
\begin{aligned}
V & =-\int_{0}^{d} E_{0} \cdot d \ell \\
& =-\int_{0}^{d} E_{0} \cos \left(180^{\circ}\right) d \ell \\
& =\int_{0}^{d} E_{0} d \ell \\
& =2 E_{b} b+E_{0}(d-2 b)
\end{aligned}
$$

The electric field in the dielectric residue, $\kappa$, is

$$
E_{b}=\frac{E_{0}}{\kappa}
$$

Therefore the voltage across the plates is

$$
\begin{aligned}
V & =E_{0}\left(\frac{2 b}{\kappa}+d-2 b\right) \\
& =E_{0}\left(d-2 b\left(1-\frac{1}{\kappa}\right)\right)
\end{aligned}
$$


To determine the effect of the dielectric, it is necessary to examine the term $\left(1-\frac{1}{\kappa}\right)$ for extreme values of $\kappa$. The lower bounds of value that any the dielectric can have is the value in vacuum $(\kappa=1)$. For air, $\kappa \approx 1$. Therefore if no dielectric film was present

$$
\left(1-\frac{1}{\kappa}\right) \approx 0
$$

For cases in which the dielectric constant is greater than 1

$$
\lim _{\kappa \rightarrow \infty}\left(1-\frac{1}{\kappa}\right)=1
$$

Therefore

$$
0 \leq\left(1-\frac{1}{\kappa}\right) \leq 1
$$

which results in the following two voltage extrema

$$
E_{0} d \leq E_{0}\left(d-2 b\left(1-\frac{1}{\kappa}\right)\right) \leq E_{0}(d-2 b)
$$

The latter extrema is that which can be used to determine the effect of the dielectric film. Using the latter extrema, the voltage across the electroplates is

$$
V=E_{0}(d-2 b) \approx E_{0} d \text { for } \mathrm{b}<<\mathrm{d} .
$$

Thus, the film has a negligible effect on the voltage across the electroplates and the capacitance across the plates.

$$
\begin{aligned}
C & =\frac{q}{V}=\frac{\varepsilon_{0} w \ell E_{0}}{E_{0}\left(d-2 b\left(1-\frac{1}{\kappa}\right)\right)} \\
& =\frac{\varepsilon_{0} w \ell}{\left(d-2 b\left(1-\frac{1}{\kappa}\right)\right)} \\
& \approx \frac{\varepsilon_{0} w \ell}{d}
\end{aligned}
$$


The sensor in Fig. 5 was used to measure the effect of residual fluid. Results of the effects are summarized in Table 2. Capacitor geometry was maintained at $22.86 \mathrm{~cm}$ by $0.32 \mathrm{~cm}$. Two plate separations were used. The plate separation for the first configuration was $0.46 \mathrm{~cm}$. The sensor was completely immersed in transmission fluid and then completely removed from the fluid with a film remaining. The frequency was measured to be $6.58 \mathrm{MHz}$. After the plates were wiped clean, the frequency was measured to be $6.63 \mathrm{MHz}$. The removal of the viscous film changed the response frequency by $0.05 \mathrm{MHz}$. The $22.86 \mathrm{~cm}$ sensor frequency ranged from $6.65 \mathrm{MHz}$ $(0.0 \mathrm{~cm}$ level) to $5.61 \mathrm{MHz}(22.86 \mathrm{~cm})$ for a difference of $1.05 \mathrm{MHz}$. A measurement error of $0.05 \mathrm{MHz}$ would equivalent to $1.07 \mathrm{~cm}$ when the container is empty.

The second sensor configuration had a plate separation of $0.16 \mathrm{~cm}$. When the second sensor was removed from the fluid, its response frequency was $5.40 \mathrm{MHz}$. The second sensor configuration has a response frequency of $5.43 \mathrm{MHz}$ when the plates were wiped. The complete range of the sensor was $1.09 \mathrm{MHz}$. The residue altered the frequency by $0.03 \mathrm{MHz}$ which would be equivalent to $0.64 \mathrm{~cm}$. The effect of film becomes more negligible as the fluid level increases because the plates have more exposure to the fluid than the film. The capillary effect was much more pronounced when the plates were separated by $0.16 \mathrm{~cm}$ than by $0.46 \mathrm{~cm}$. The larger separation allowed the fluid to drain from between the plates more rapidly.

\begin{tabular}{|c|c|c|}
\hline Event & $\begin{array}{l}0.46 \mathrm{~cm} \text { Plate } \\
\text { Separation }\end{array}$ & $\begin{array}{l}0.16 \mathrm{~cm} \text { Plate } \\
\text { Separation }\end{array}$ \\
\hline Frequency at $0.0 \mathrm{~cm}$ fluid level & $6.66 \mathrm{MHz}$ & $5.45 \mathrm{MHz}$ \\
\hline $\begin{array}{l}\text { Frequency when immersed in } \\
22.86 \mathrm{~cm} \text { fluid level }\end{array}$ & $5.61 \mathrm{MHz}$ & $4.36 \mathrm{MHz}$ \\
\hline Change in frequency & $1.05 \mathrm{MHz}$ & $1.09 \mathrm{MHz}$ \\
\hline $\begin{array}{l}\text { Frequency when sensor is } \\
\text { removed from fluid but with } \\
\text { residual fluid }\end{array}$ & $6.58 \mathrm{MHz}$ & $5.40 \mathrm{MHz}$ \\
\hline $\begin{array}{l}\text { Frequency when sensor residual } \\
\text { fluid is wiped clean }\end{array}$ & $6.63 \mathrm{MHz}$ & $5.43 \mathrm{MHz}$ \\
\hline Frequency error due to fluid & $0.05 \mathrm{MHz}$ & $0.035 \mathrm{MHz}$ \\
\hline
\end{tabular}

Table 2 Frequency error from residual fluid. 


\section{Concluding Remarks}

A fluid-level sensor and measurement acquisition system have been presented that allow the level measurement of any substance that can be placed within capacitance plates. The method of applying power to the sensor, a capacitor-inductor circuit, and acquiring the measurement is done without physical contact to a power source or to data acquisition equipment. The measurement acquisition method uses a series of sequential magnetic field harmonics. For each harmonic, the system transmits a harmonic magnetic field that produces current in the sensor via Faraday induction. Once electrically active, the sensor produces a magnetic field. The response frequency of the magnetic field is dependent upon the amount of sensor capacitor surface area exposed to the dielectric substance. The measurement system switches from transmitting a harmonic magnetic field to receiving the magnetic field produced by the sensor. The amplitude of the received response is compared to previous responses to ascertain if the measurement system has detected a response inflection. The "transmit-receive-compare" of sequential harmonics is repeated until the inflection is identified. The harmonic producing the amplitude inflection is the sensor resonant frequency.

Analytic models for sensor design and sensor sensitivity to key design parameters were presented. The models were developed for two stratified dielectrics. The parameters included sensor geometric properties and fluid dielectric properties. The models will allow designers to create desired sensor sensitivity and frequency range. The sensor resonant frequency corresponds to the interface of the two stratified dielectric fluent substances such as fuel and air.

Measurements of various substance levels were presented to demonstrate the functionality of the sensor. The substances dielectric properties were constant. Results of using two sensor designs were presented. One sensor was designed to allow its geometry to be varied to quantify measurement sensitivity to sensor geometry. The sensor design demonstrated that capacitor plates could be fabricated from copper foil and potentially from using metal deposition methods. Metal deposition techniques will result is sensors of lighter weight. The other sensor design was used to demonstrate the range of substances that could be measured. Its material properties were resilient to cryogenic temperatures and petroleum. The substances measured were a viscous liquid, a non-viscous cryogenic liquid (nitrogen), a powder and a granular substance. Measurements of liquid nitrogen fluid level demonstrated the potential for using fluid-level sensors of similar designs for other liquid cryogens such as hydrogen or oxygen.

A conceptual model demonstrated that measurements could be acquired without the sensor's electrical components being in physical contact with the dielectric substance that is being measured. Measurement applications could include caustic fluids or measuring substances in environments that could destroy electrical components. This was achieved by completely encasing all sensor components. The capacitors plates can be encased in a material that is resilient to the dielectric for which it is to be immersed. The encasing material could be a chemically resilient plastic for acids, a material capable of sustained cryogenic exposure for liquid nitrogen, hydrogen, helium or oxygen, a material of low thermal conduction for molten metals or containing a large number of hydrogen nuclides for radioactive environments. The measurement acquisition system is capable of providing power to the sensor and acquiring the 
measurement from the sensor while it is completely encased. A thin dielectric can be placed on the capacitor plates with negligible change to the sensors overall capacitance. The casing shifts the frequency range. Results presented in this paper have demonstrated that the inductor can be encased and if necessary hermetically sealed. Therefore, the entire sensor could be encased and hermetically sealed.

The effect of residual viscous fluid on measurement error was also presented. The error was demonstrated to be negligible for very thin films. Measurements using two plate separations were used to quantify the effect of residual fluid. For each configuration, the sensors were immersed in transmission fluid and then removed. The sensor frequencies when removed from fluid were compared to the frequencies measured after the sensor was wiped clean. The difference in frequency was the measurement error as a result of the residual fluid. The maximum measurement error for both configurations was $1.07 \mathrm{~cm}$ for the sensor having a length of $22.86 \mathrm{~cm}$. The error is that of the sensor not immersed in fluid. Once the sensor is partially immersed in the fluid, the effect of the residual fluid is negligible. The results demonstrated that the capacitance is altered slightly but the error is acceptable for many applications when indications and warnings are the objective of the sensor (e.g., tanks storing hydraulic fluids).

Acknowledgements The authors wish to acknowledge the contributions of Mr. Robert L. Fox (deceased), Mr. Johnny C. Mau, Dr. Qamar A. Shams, Ms. Rosa C. Webster and Ms. Robin W. Edwards, Mr. Glenn R Slayton and Mr. Gary D. Robbins of NASA Langley Research Center; Mr. P. Van Bergen; Mr. R. Campbell of Morehouse College and Georgia Institute of Technology.; NASA summer student -Mr. N. Pandey, Mr. J. Hill and Ms. S. Wright; and, Mr. K. Chandler, Mr. G. Minnes, Mr. G. Ataman, Mr. K Schmidt and Mr. R. Lavigne of Messier Dowty Inc. 


\section{References}

${ }^{1}$ Sakharov, V. E., Kuznetsov, S. A., Zaitsev, B. D., Kutnetsova, I. E., and Joshi, S. G., "Fluid Level Sensor Using Ultrasonic Lamb Waves” Ultrasonics, Vol 4, pp 319-322, 2003.

${ }^{2}$ Vazquez, C., Gonzalo, A. B., Vargas, S., and Montalvo, J., "Multi-Sensor System Using Plastic ptical Fibers for Intrinsically Safe Level Measurement," Sensors and Actuators A, Vol. 116, 2004.

${ }^{3}$ Kersey, A. D., and Dandrige, A., "Application of Fiber-Optic Sensors," IEEE Transactions on Components, Hybrids, and Manufacturing Technology, Vol 13, No. 1, March 1990.

${ }^{4}$ Golnabi, Hossein, "Design and Operation of a Fiber Optic Sensor for Fluid Level Detection," Optics and Lasers in Engineering, Vol. 41, pp 801-812 (2004).

${ }^{5}$ Raatikeinen, P., Kassamakov, I., Kakanakov, R., and Luukkala, M., "Fiber-Optic Fluid Level Sensor," Sensors and Actuators A, Vol 58, pp 93-97 (1997).

${ }^{6}$ Wang, A., Gunther, M. F., Murphy, K. A., and Claus, R. O., "Fiber-Optic Fluid Level Sensor," Sensors and Actuators A, Vol 35, pp 161-164 (1992).

${ }^{7}$ Woodard, S. E., Taylor, B. D. , Shams, Q. A. and Fox, R. L., "Magnetic Field Response Measurement Acquisition System," NASA Technical Memorandum 2005-213518, February 2005.

${ }^{8}$ Woodard, S. E., Taylor, B. D., Shams, Q. A., Fox, R. L. and Bryant, R. G.,"Magnetic Field Response Measurement Acquisition System," U. S. Patent Application Number 10/839,445, April 30, 2004.

${ }^{9}$ Woodard, S. E. and Taylor, B. D., "Magnetic Field Response Sensor for Conductive Media," Submitted to U. S. Patent Application Number 10/839,448, April 30, 2004.

${ }^{10}$ Woodard, S. E., Taylor, B. D., Shams, Q. A. and Fox, R. L., "Magnetic Field Response Measurement Acquisition System" submitted to Measurement Science and Technology, Aug 2004.

${ }^{11}$ Woodard, S. E., Taylor, B. D., Shams, Q. A., and Fox, R. L., "L-C Measurement Acquisition Method For Aerospace Systems," Proceedings of the 2003 AIAA Aviation Technology, Intergration and Operations Technical Fourm, AIAA Paper No. 2003-6842, Denver,CO, November 17-19, 2003.

${ }^{12}$ Taylor, B. D. and Woodard, S. E., "Wireless Fluid Level Measuring System," Submitted to U. S. Patent Application Number, September 12, 2005. LAR 17155.

${ }^{13}$ Halliday, D., and Resnick, R. "Physics Part Two," John Wiley and Sons, New York, NY, 1978, pp.650-665 and 770-888.

${ }^{14}$ Lorrain, P. and Corson, D., "Electromagnetic Fields and Waves," W. H. Freeman and Company, 1970, San Francisco, CA, pp. 91-128, and 292-373

${ }^{15}$ Carreau, Mark, "Wire Damage Discovered in All Four Space Shuttles," Houston Chronicle, Aug 16, 1999

${ }^{16}$ Ray, Justin, "Wiring Review Delays Delta 2 Launch of Air Force Satellite," Spaceflight Now, July 25, 2002.

${ }^{17}$ National Transportation Safety Board, “Aircraft Accident Report: In-flight Break-up Over the Atlantic Ocean, Trans World Airlines Flight 800, Boeing 747-131, N93119 Near East Moriches, New York, July 17, 1996" NTSB/AA-00/03, DCA96MA070, PB2000-910403, Notation 6788G, Aug 23, 2000.

${ }^{18}$ Transportation Safety Board of Canada, “Aviation Investigative Report: In-Flight Fire Leading to Collision with Water, Swissair Transport Limited, McDonnell Douglas MD-11 HB-IWF, 
Peggy's Cover, Nova Scotia 5nm SW, 2 September 1998" Report Number A98H0003.

${ }^{19}$ Konchin, B., Slavik, I. and Coery, R. W., "Fluid Sensing System," U. S. Patent 6, 335, 690, Jan. 2002.

${ }^{20}$ Fonseca, M. A., English, J. M., Arx, M. V. Allen, M. G., "High Temperature Characterization of Ceramic Pressure Sensors," Proceedings of 1999 IEEE MEMS Workshop, pp 146-149.

${ }^{21}$ Allen, M. A., and English, J. M., "System and Method for the Wireless Sensing of Physical Properties," U. S. Patent 6, 11,520, Aug. 2000. 


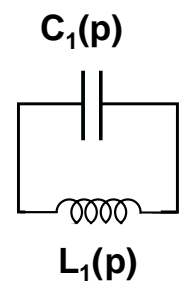

Sensor 1

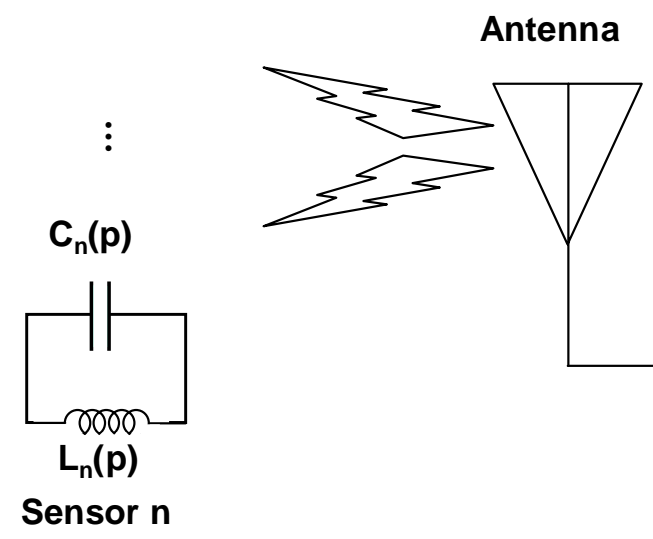

Measurement Acquisition System

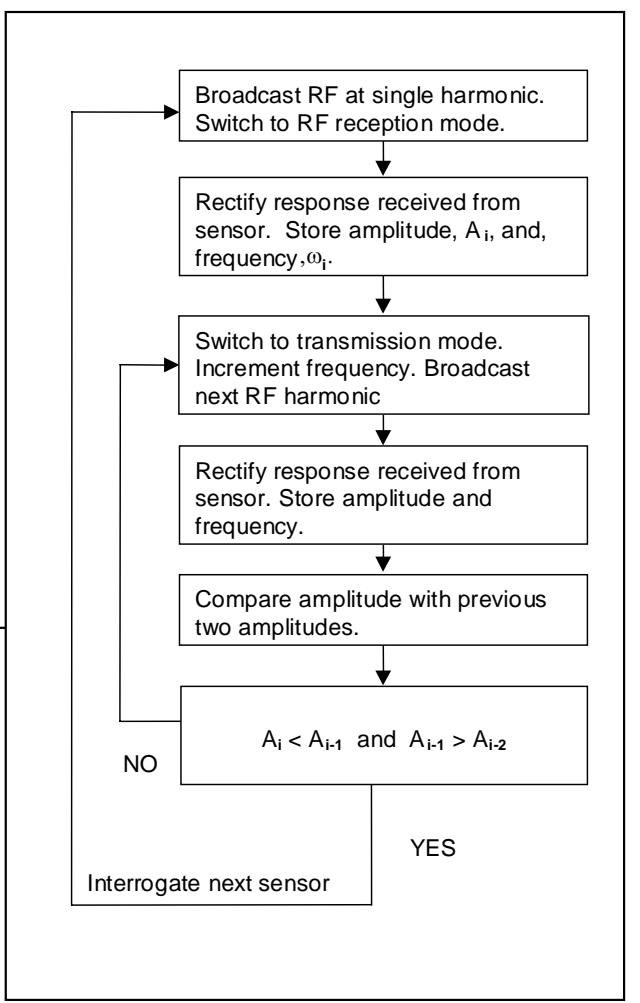

Fig. 1. Schematic of magnetic field response measurement acquisition method 


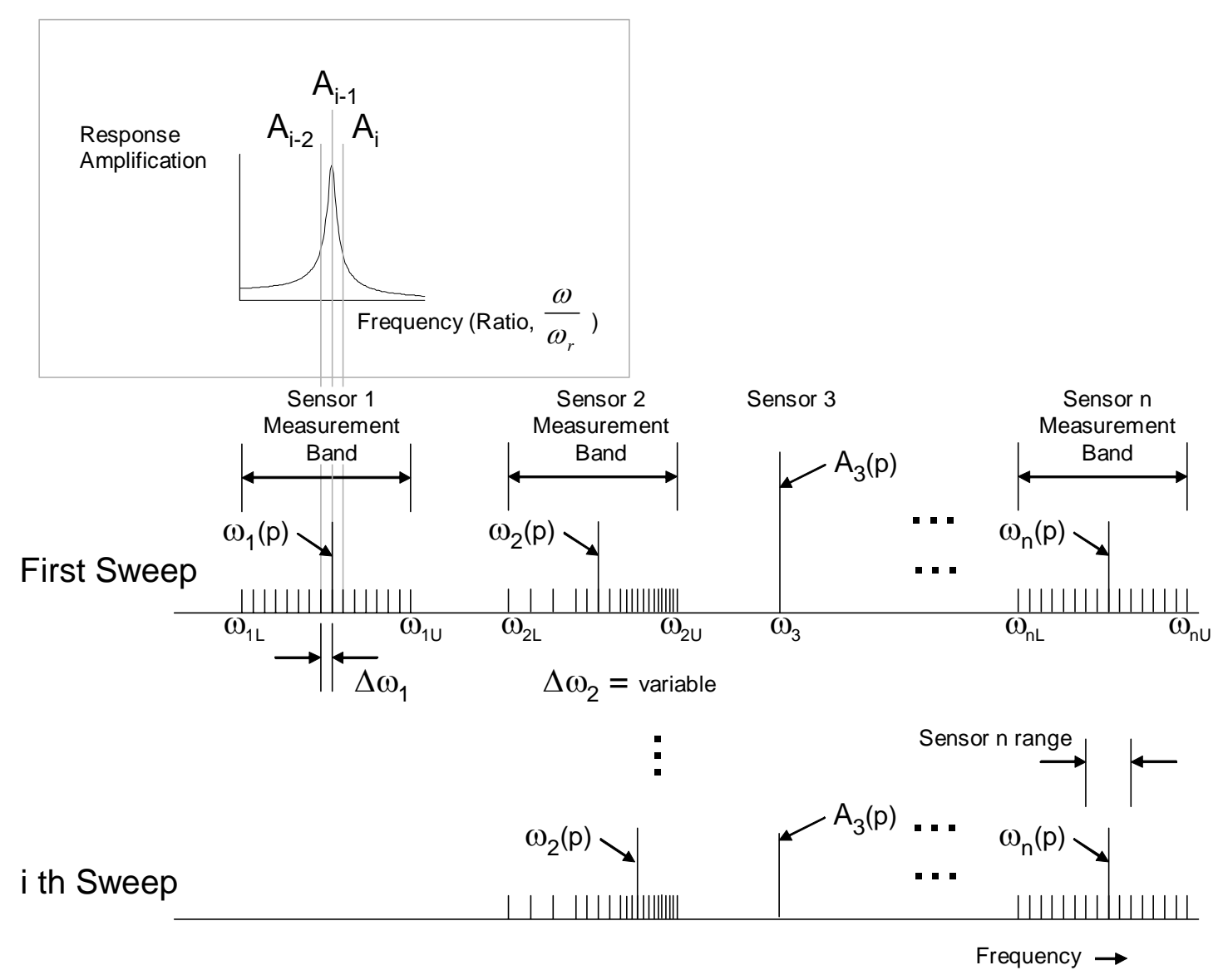

Fig. 2 Magnetic field response sensor measurement bands and resolution during successive frequency sweeps.

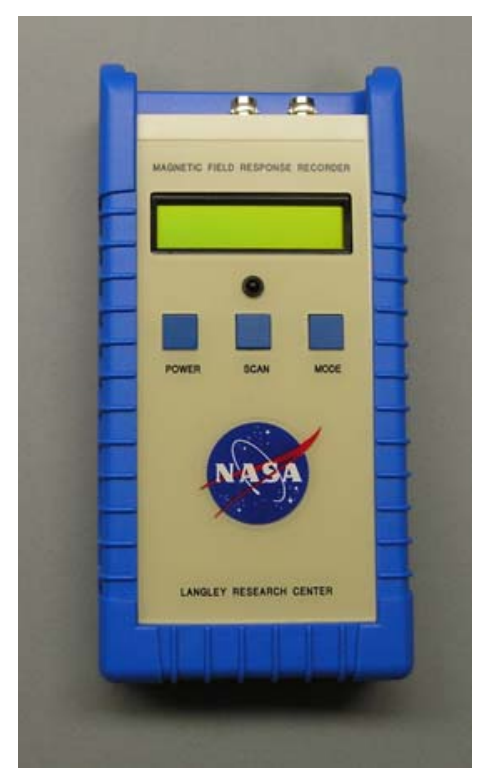

Fig. 3 Portable magnetic field response recorder 


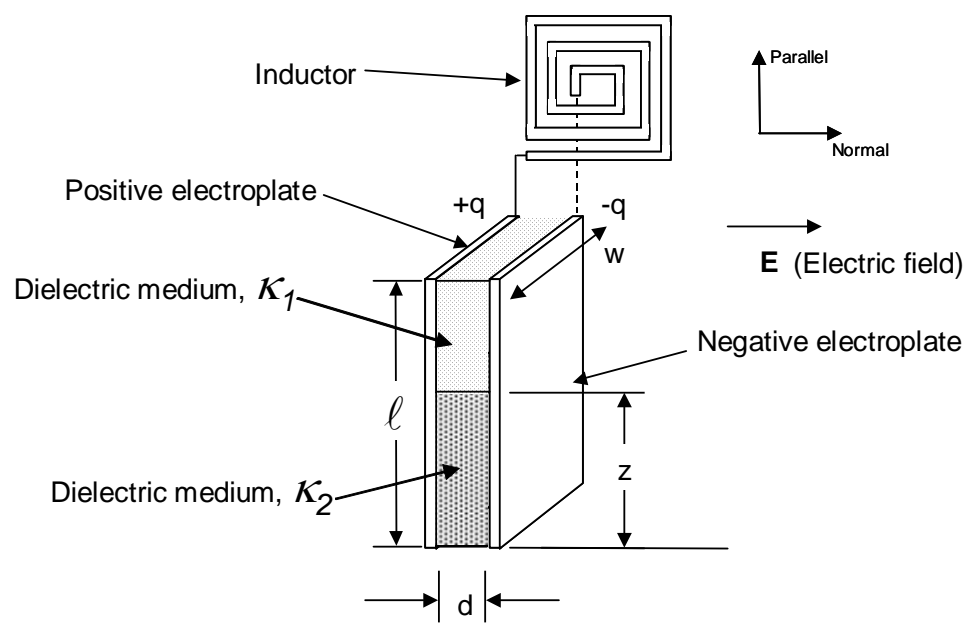

Fig. 4 Capacitor with negative and positive stationary electroplates partially placed into a dielectric medium. The dielectric medium has dielectric constant, $\kappa$. The electroplates are electrically coupled to an inductor in parallel.

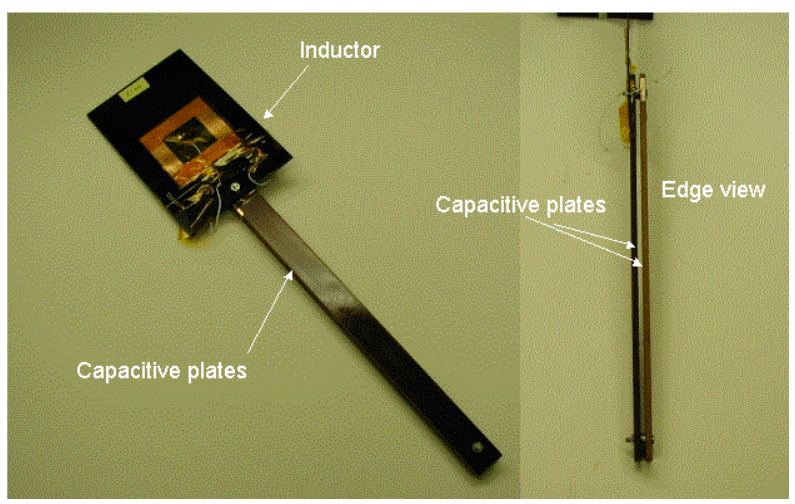

Fig. 5 Magnetic field response fluid-level sensor 


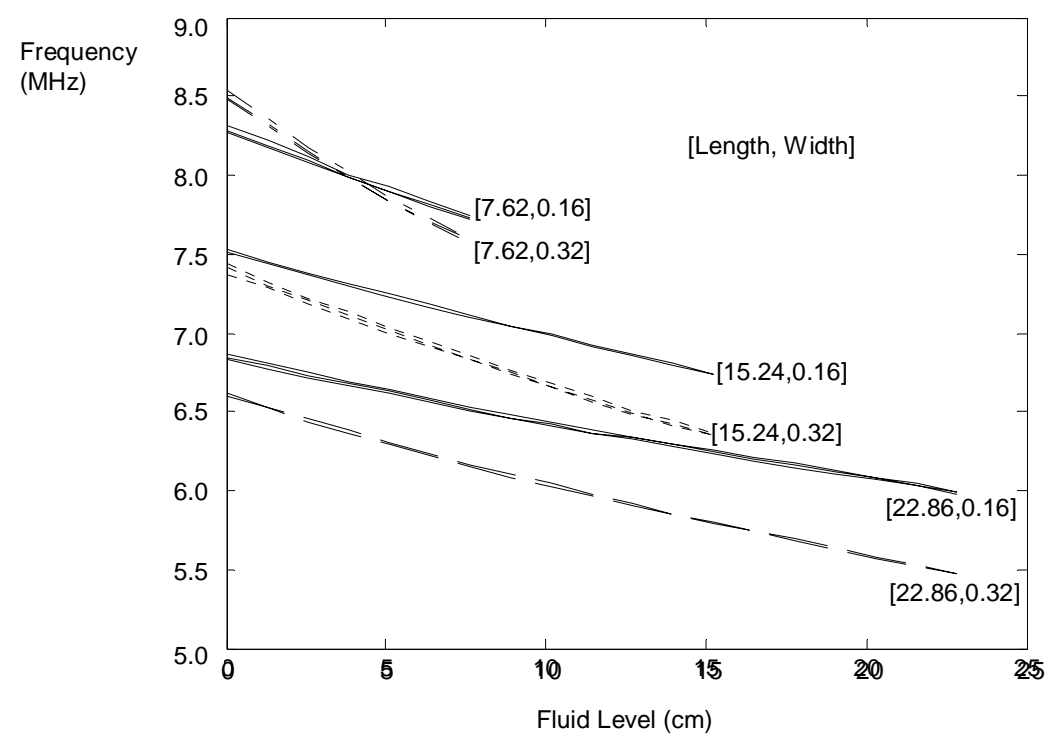

Fig. 6 Magnetic field response sensitivity to sensor geometry with fluid level as measured by interrogation antenna

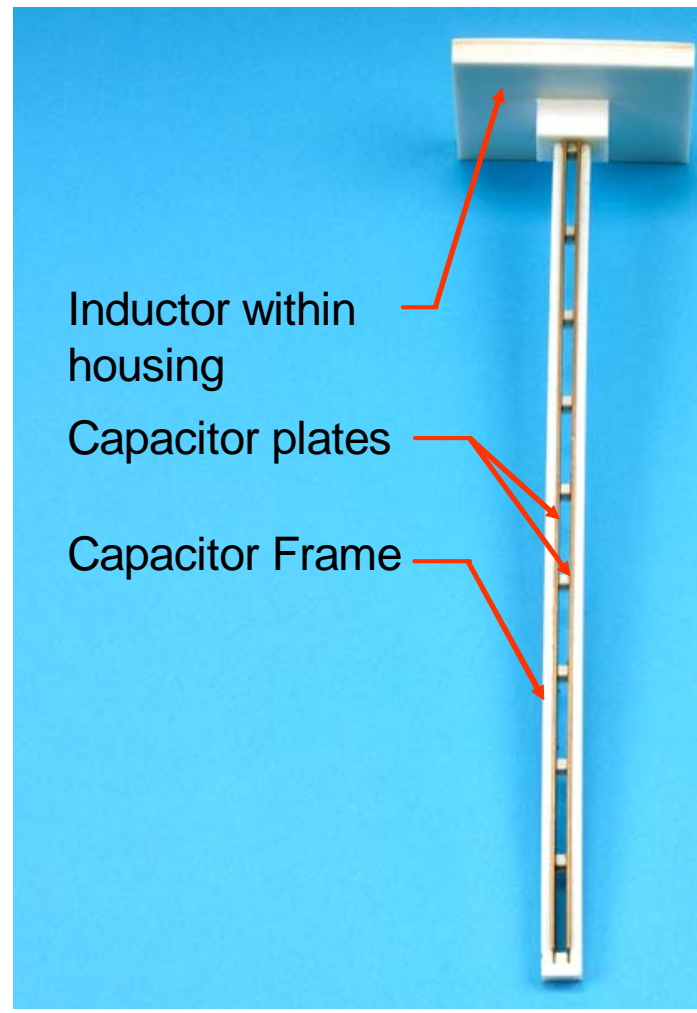

a. Magnetic field response fluid level sensor

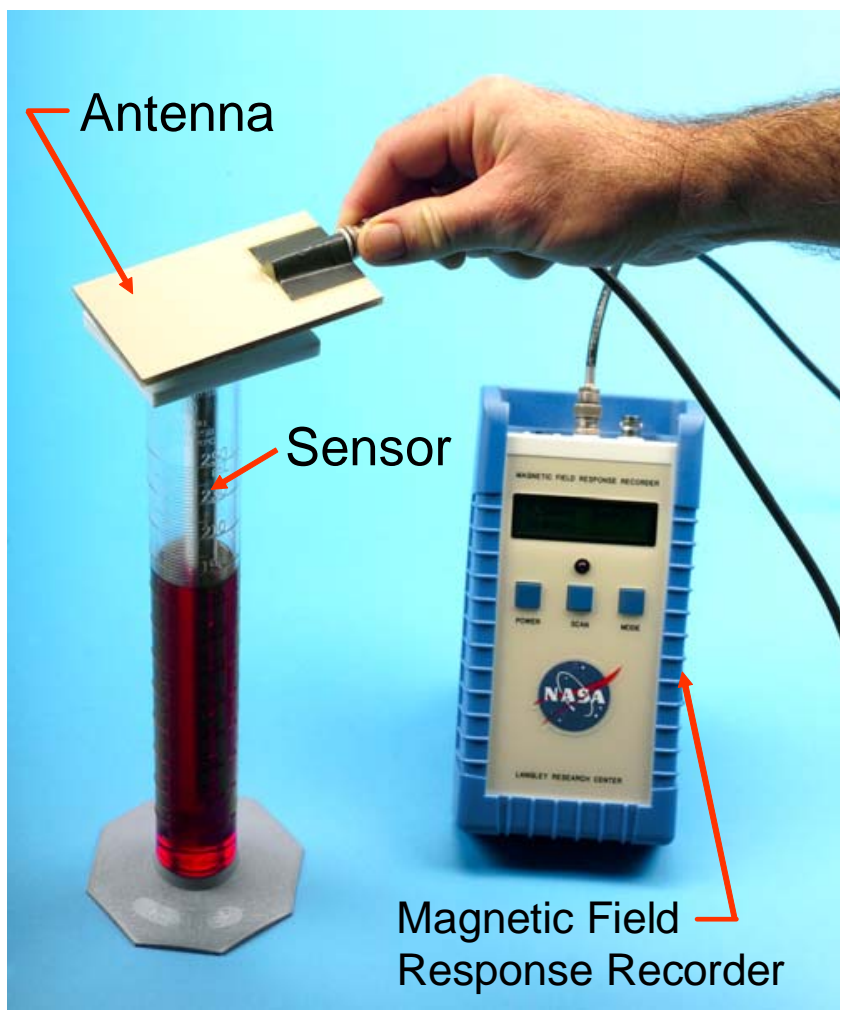

b. Sensor being interrogated using magnetic response field recorder.

Fig. 7 Electrically passive sensor for measuring dielectric levels such as liquids and magnetic field response recorder for interrogation any magnetic field response sensor 


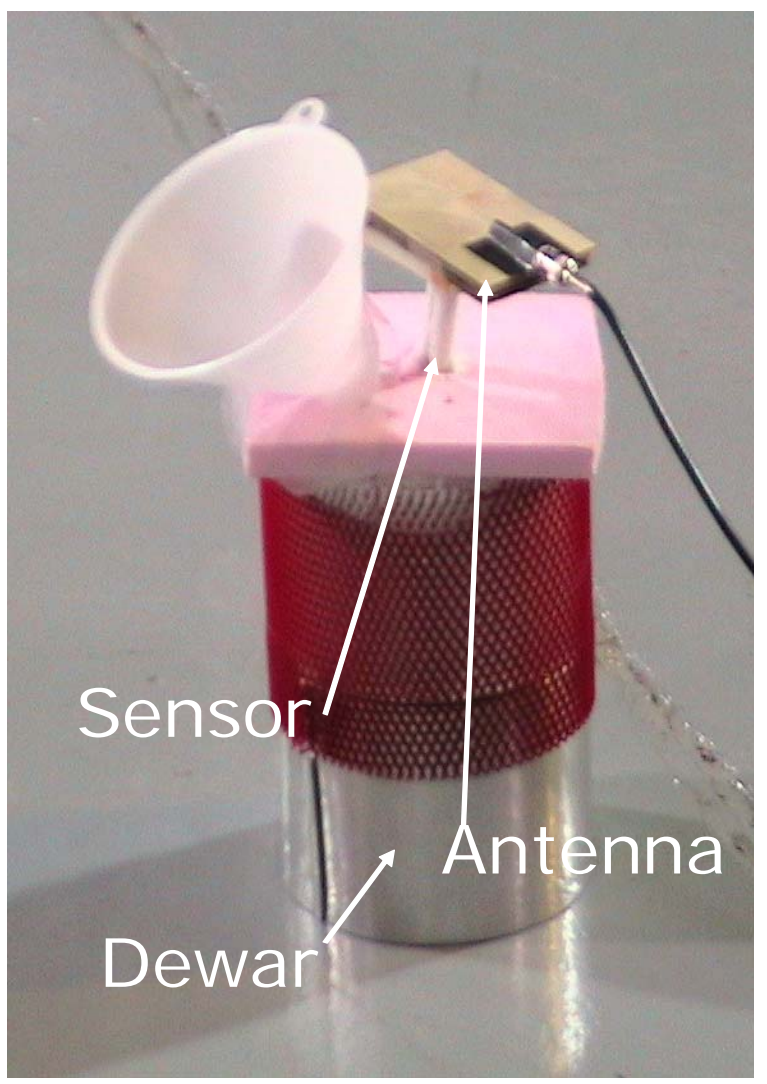

Fig. 8 Measurement of liquid nitrogen level

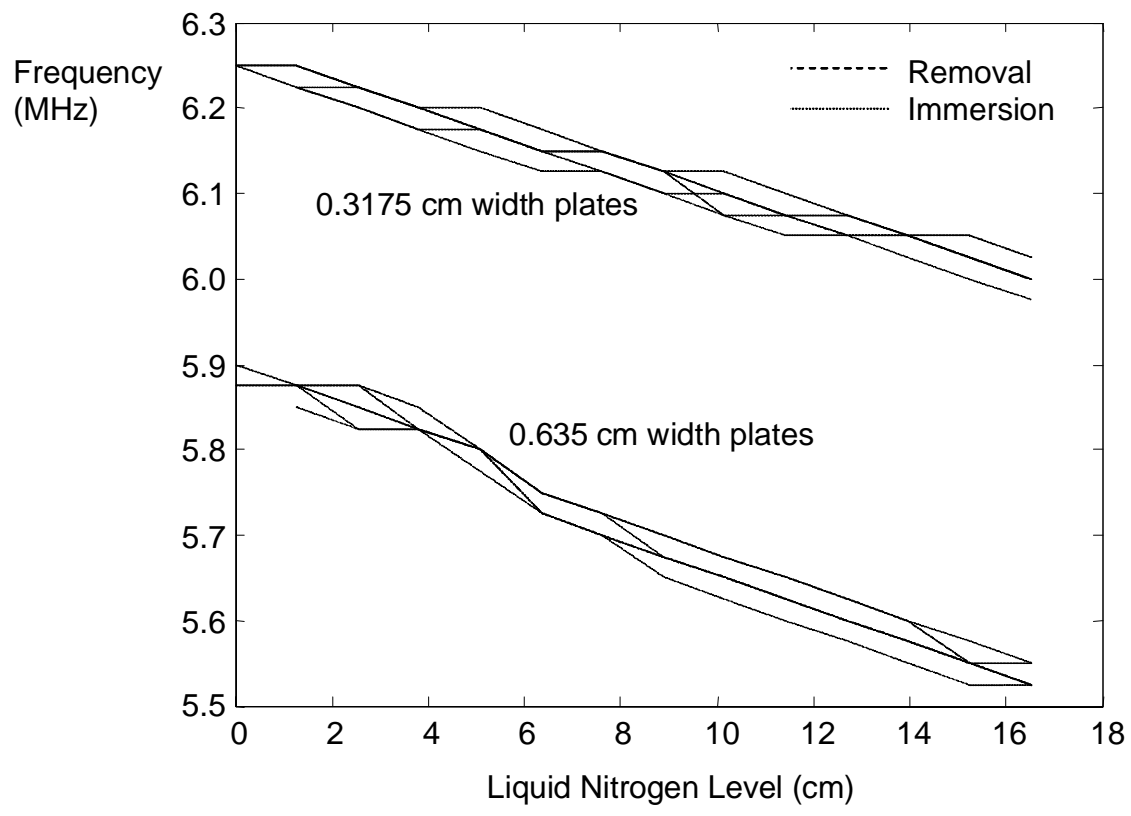

Fig. 9 Magnetic field response frequency dependency upon immersion level in liquid nitrogen 


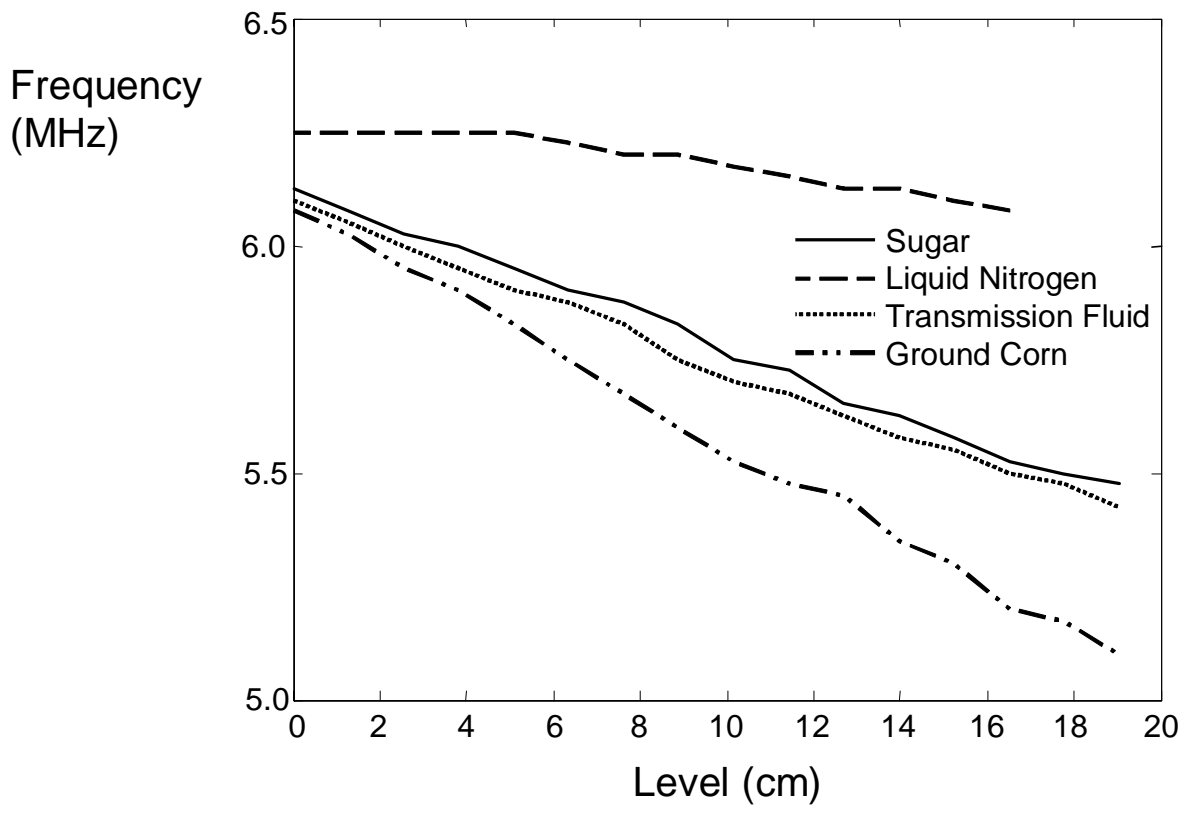

Fig. 10 Measurements of various dielectric substance levels using a magnetic field response fluid level sensor with Ertalyte PET-P support frame for capacitor plates and inductor housing.

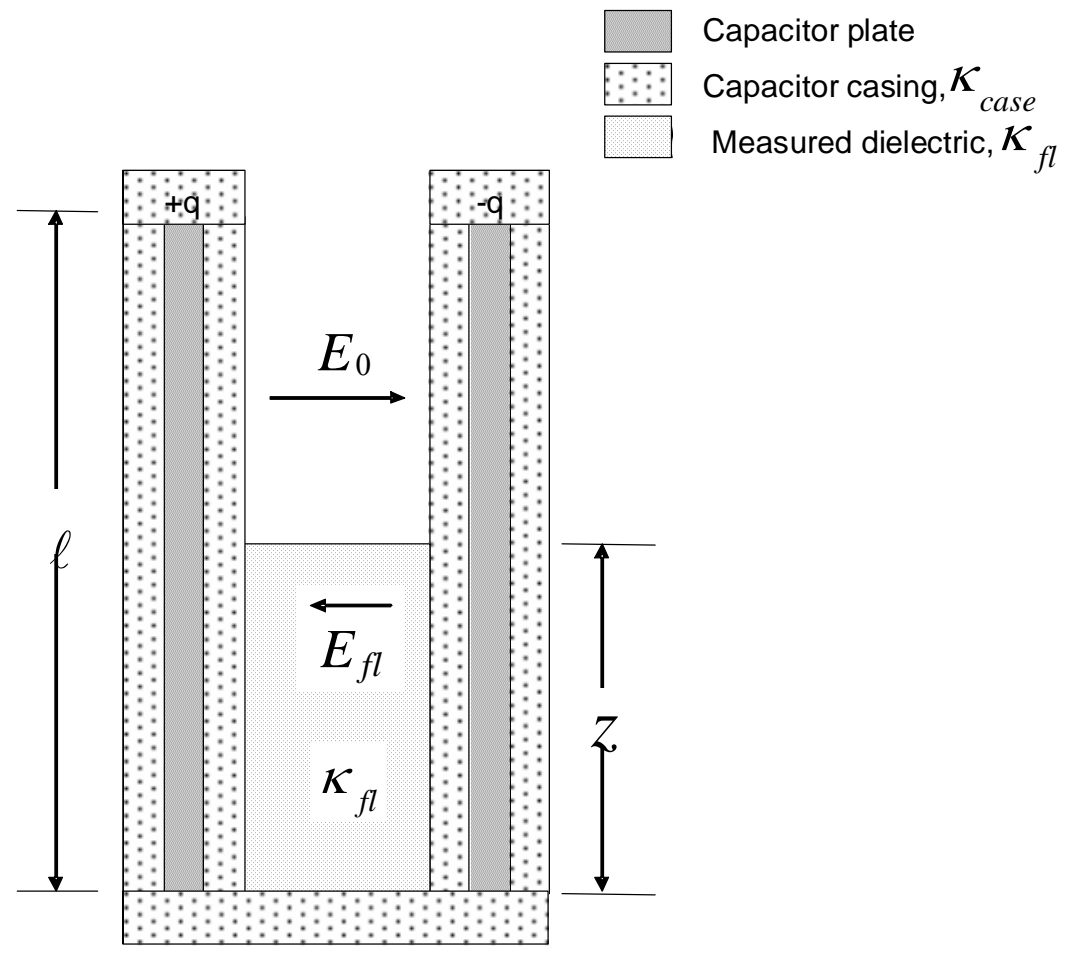

Fig. 11 A measured dielectric between two capacitor plates that have been encapsulated in an electrically non-conductive casing. 


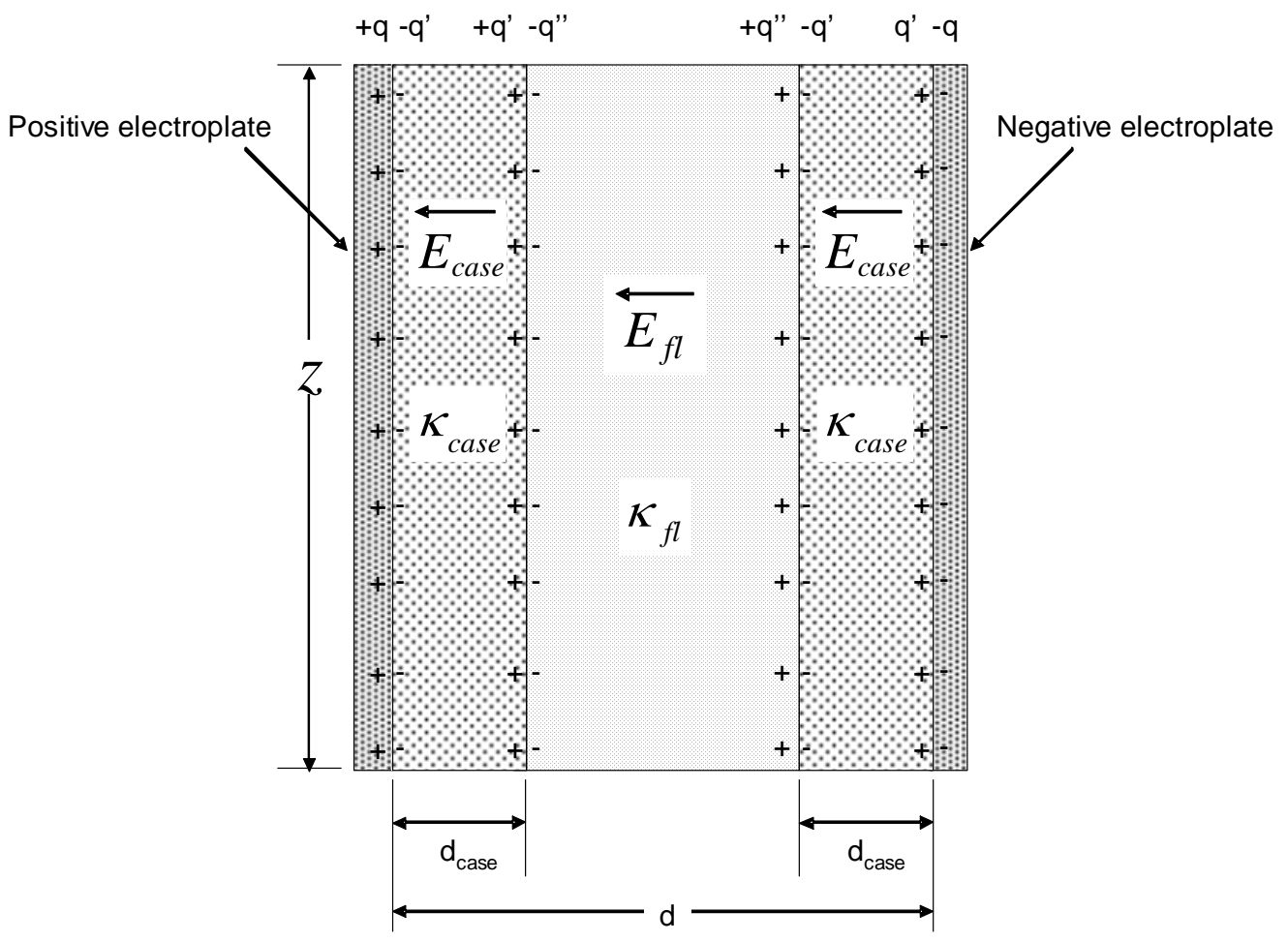

Fig. 12 A measured dielectric, $\kappa_{f l}$, between two thin dielectrics, $\kappa_{\text {case }}$.

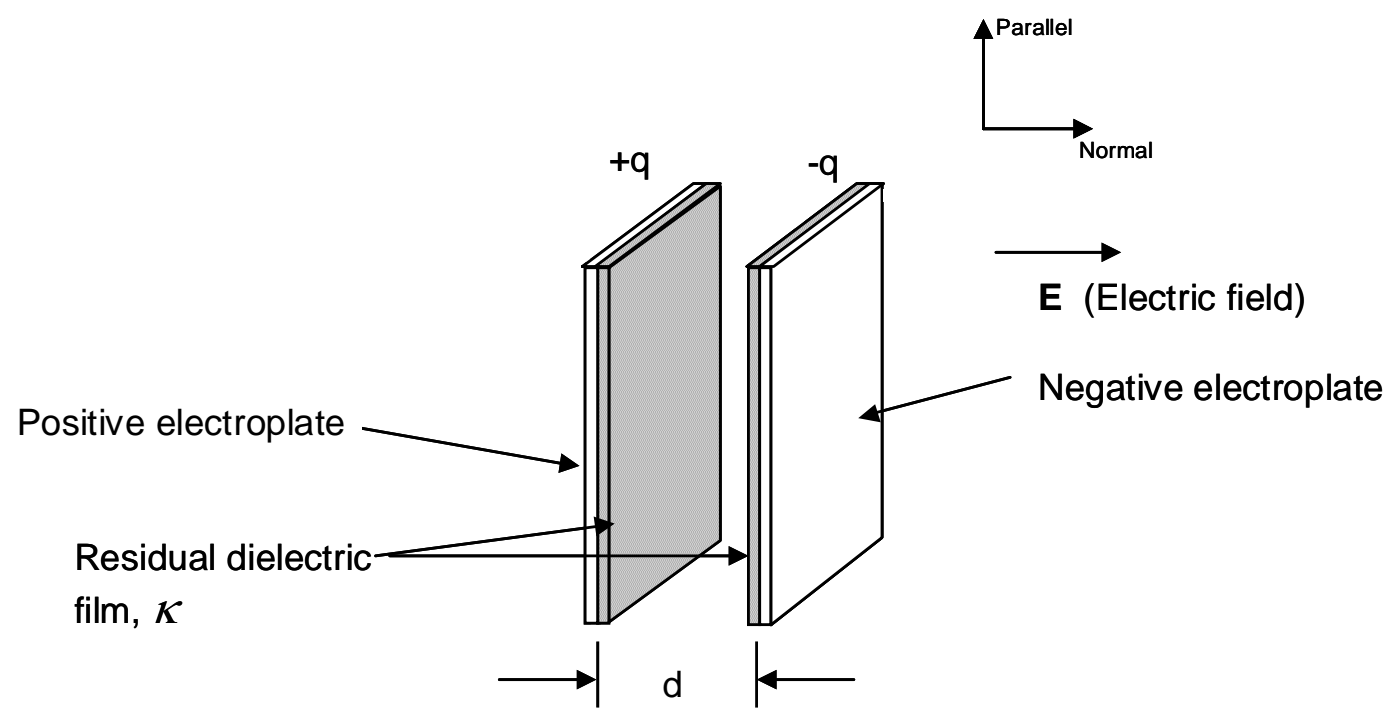

Fig 13. Residual dielectric remaining on electroplates as thin film. 


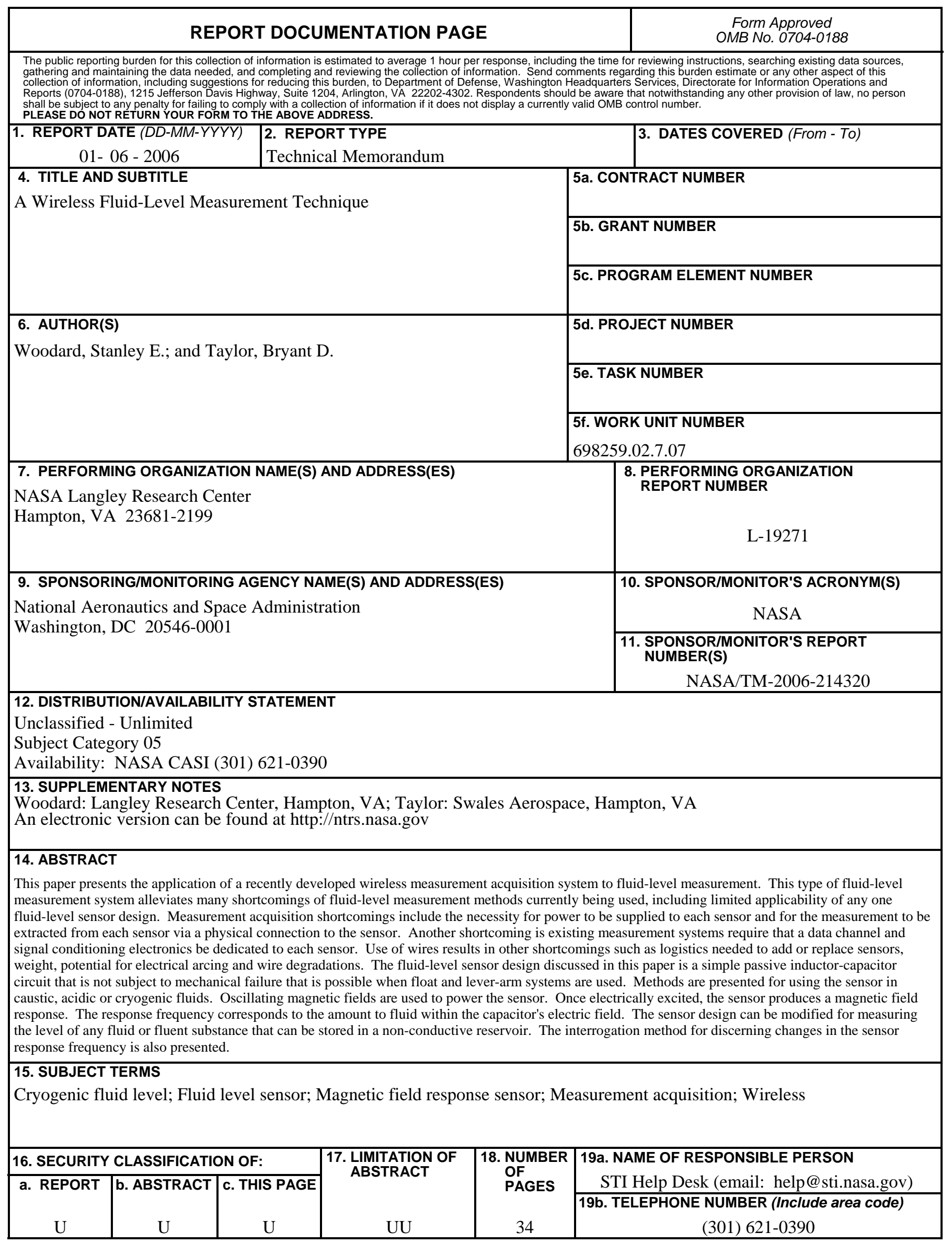

